Bull. Korean Math. Soc. 50 (2013), No. 4, pp. 1069-1077

http://dx.doi.org/10.4134/BKMS.2013.50.4.1069

\title{
SOME ALGEBRAIC AND TOPOLOGICAL PROPERTIES OF THE NONABELIAN TENSOR PRODUCT
}

\author{
Daniele Ettore Otera, Francesco G. Russo, and Corrado Tanasi
}

\begin{abstract}
Several authors investigated the properties which are invariant under the passage from a group to its nonabelian tensor square. In the present note we study this problem from the viewpoint of the classes of groups and the methods allow us to prove a result of invariance for some geometric properties of discrete groups.
\end{abstract}

\section{Formulation of the problem in terms of class operators}

Brown and others wrote two fundamental contributions $[4,5]$ on the so-called nonabelian tensor square of a group, leaving a series of open questions which have been successively discussed in $[6,7,9,10,11,12,15,16,19,20]$ and in other works of different authors in recent years.

Following [4, 5], one says that two groups $G$ and $H$ act compatibly upon each other, if

$$
{ }^{g} h g^{\prime}=g\left({ }^{h}\left({ }^{g^{-1}} g^{\prime}\right)\right), \quad{ }^{h} h^{\prime}={ }^{h}\left({ }^{g}\left({ }^{h^{-1}} h^{\prime}\right)\right)
$$

for $g, g^{\prime} \in G$ and $h, h^{\prime} \in H$, and if they act upon themselves by conjugation. Note that we write conjugation on the left, so that ${ }^{g} g^{\prime}=g g^{\prime} g^{-1}$ and ${ }^{g} g^{\prime} g^{\prime-1}=$ $\left[g, g^{\prime}\right]$. The nonabelian tensor product $G \otimes H$ of $G$ and $H$ is then the group generated by the symbols $g \otimes h$ with defining relations

$$
g g^{\prime} \otimes h=\left({ }^{g} g^{\prime} \otimes{ }^{g} h\right)(g \otimes h), \quad g \otimes h h^{\prime}=(g \otimes h)\left({ }^{h} g \otimes{ }^{h} h^{\prime}\right) .
$$

If $G$ and $H$ act trivially upon each other, then $G^{a b} \otimes_{\mathbb{Z}} H^{a b}$ is the usual abelian tensor product (see [5, Proposition 2.4]) of the abelian groups $G / G^{\prime}$ and $H / H^{\prime}$. On the other hand, if $G=H$ and all actions are conjugation, then the group $G \otimes G$ is called the nonabelian tensor square of $G$. Some of the main rules of

\footnotetext{
Received January 17, 2012.

2010 Mathematics Subject Classification. Primary 20J99; Secondary 57M50.

Key words and phrases. nonabelian tensor product, actions of groups, quasi simple filtration, homology, homotopy group.

The first author was supported by the European Commission's Marie Curie IntraEuropean Fellowship n. PIEF-GA-2009-236306. The second author thanks Ibnu Sina Institute (Universiti Teknologi Malaysia) for a partial support of the present research.
} 
calculation can be found in [4, Propositions 1, 2, and 3]. In particular, it turns out that the map

$$
\kappa: g \otimes h \in G \otimes H \mapsto \kappa(g \otimes h)=[g, h] \in D_{H}(G)
$$

is an epimorphism of groups, where

$$
D_{H}(G)=\left\langle{ }^{g} h h^{-1} \mid g \in G, h \in H\right\rangle=[G, H]
$$

is the derivative of $G$ by $H$ (see [9, p. 22] or [19, Definition 2.1]). Analogously, one may define the derivative $D_{G}(H)$ of $H$ by $G$, finding that $D_{G}(G)=[G, G]$ and $D_{H}(H)=[H, H]$ are the usual derived subgroups of $G$ and $H$, respectively. Proposition 2.3 of [19] shows that $D_{H}(G)$ is a normal subgroup of $G$, fixed under the action of $H$, that is, $D_{H}(G)^{H}=D_{H}(G)$ (whenever $G$ and $H$ act compatibly upon each other) and $G / D_{H}(G)$ is the largest quotient of $G$ on which $H$ acts trivially. The same happens for $D_{G}(H)$.

The nonabelian exterior product $G \wedge H$ of $G$ and $H$ is the group

$$
G \wedge H=(G \otimes H) / \nabla(G \cap H),
$$

where $\nabla(G \cap H)=\langle g \otimes g \mid g \in G \cap H\rangle$ turns out to be a central subgroup of $G \otimes H$. It is easy to check that

$$
\kappa^{\prime}: g \wedge h \in G \wedge H \mapsto \kappa^{\prime}(g \wedge h)=[g, h] \in D_{H}(G)
$$

is a well-defined epimorphism of groups. If $G=H$ and all actions are conjugation, then $G \wedge G$ is called the nonabelian exterior square of $G$.

For the convenience of the reader, we recall the commutative diagram with exact rows and central extensions as columns as in [4]. It involves the second homology group of $G, H_{2}(G)=H_{2}(G, \mathbb{Z})=\operatorname{ker} \kappa^{\prime}$, which is isomorphic to the Schur multiplier $M(G)$ of $G$, the third homology group $H_{3}(G)$ of $G$, the Whitehead's quadratic functor $\Gamma$ and $\operatorname{ker} \kappa=J_{2}(G)$ (see $[4,5]$ for details):

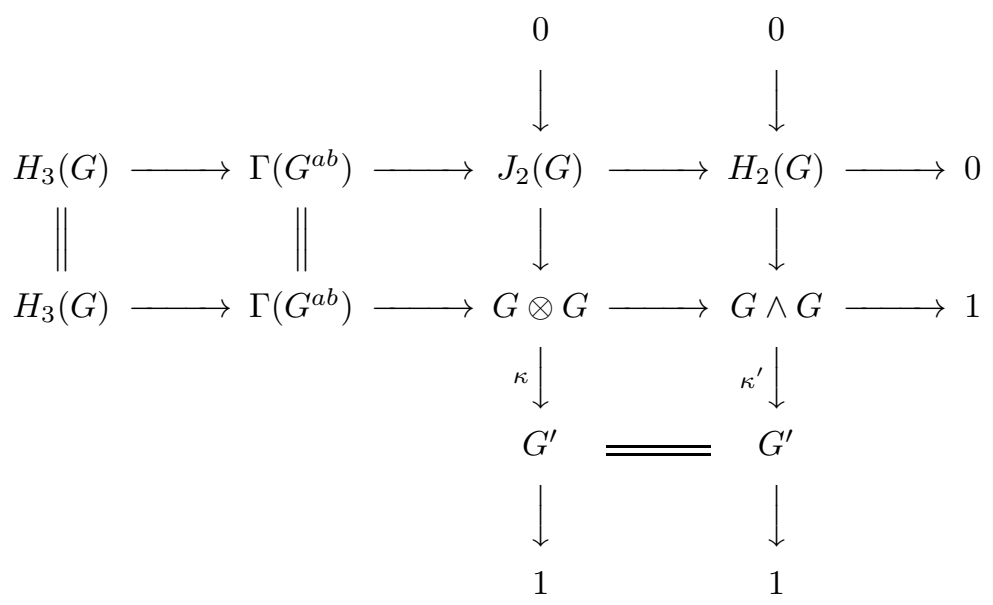

From (1.1), one sees that the structure of $G$ is influenced by that of $G \otimes G$ and viceversa. This motivated many authors to study those properties of $G$ that 
are invariant under the operation $G \otimes G$. More technically, given a class of groups $\mathfrak{X}$, the following implication

$$
\text { If } G \in \mathfrak{X} \text {, then } G \otimes G \in \mathfrak{X}
$$

has been studied for several choices of $\mathfrak{X}$. With the notation $\mathfrak{X}=\mathrm{S} \mathfrak{X}$, we mean that $\mathfrak{X}$ is closed with respect to forming subgroups and with $\mathfrak{X}=Q \mathfrak{X}$ that $\mathfrak{X}$ is closed with respect to forming quotients. The class operator NT is defined by

$$
\mathrm{NTX}=(G \mid G \otimes G \in \mathfrak{X}) .
$$

It is easy to see that NTX is well-defined and that the definition of $G \otimes G$ involves the existence of an action of $G$ on itself by conjugation and in a compatible way. Now it is clear that (1.2) is equivalent to the closure of $\mathfrak{X}$ with respect to $\mathrm{NT}$, that is, $\mathrm{NT} \mathfrak{X}=\mathfrak{X}$. Counterexamples may be given, once $\mathfrak{X}$ does not satisfy elementary closure properties such as $\mathfrak{X}=\mathrm{S} \mathfrak{X}=\mathrm{Q} \mathfrak{X}$. For instance, let $\mathbb{Z}_{2}$ be the cyclic group of order $2, \mathbb{Z}_{4}$ the cyclic group of order 4 and $D_{8}$ the dihedral group of order 8; then [4, Table I, line 9] shows that $\left(\mathbb{Z}_{4} \times D_{8}\right) \otimes\left(\mathbb{Z}_{4} \times D_{8}\right)=\mathbb{Z}_{2}^{8} \times \mathbb{Z}_{4}$. We conclude that the property of being factorized in the direct product of an abelian group by a nonabelian group is not closed with respect to NT. But also the property of being a cyclic group, which is closed with respect to $\mathrm{S}$ and $\mathrm{Q}$, may give problems; the nonabelian tensor product of two cyclic groups may not be cyclic necessarily.

We recall now some notations from $[14,16,17]$ :

$\check{\mathfrak{C}}$ is the class of all Černikov groups;

$\mathfrak{E}_{n}$ is the class of all $n$-Engel groups $(n \geq 1)$;

$\mathfrak{F}$ is the class of all finite groups;

$\mathfrak{F}_{p}$ is the class of all finite $p$-groups ( $p$ prime);

$\mathfrak{N}$ is the class of all nilpotent groups;

$\mathfrak{P}$ is the class of all periodic groups;

$\mathfrak{S}$ is the class of all soluble groups;

$\mathfrak{S}_{2}$ is the class of all soluble minimax groups;

$\mathfrak{S}_{2} \mathfrak{F}$ is the class of all (soluble minimax)-by-finite groups;

$\mathfrak{Z}^{\infty}$ is the class of all polycyclic groups;

$\mathfrak{Z}^{\infty} \mathfrak{F}$ is the class of all polycyclic-by-finite groups.

The state of knowledge on NT is summarized by the next result, where we refer, directly or indirectly, to contributions of Bacon, Blyth, Ellis, Kappe, Inassaridze, Moravec, Morse, Nakaoka, Rocco, Russo, Sarmin, Visscher.

Theorem 1.1 (See $[12,16,19,20])$. The class operator NT is closed with respect to $\check{\mathfrak{C}}, \mathfrak{E}_{2}, \mathfrak{F}, \mathfrak{F}_{p}, \mathfrak{N}, \mathfrak{Z}^{\infty}, \mathfrak{S}, \mathfrak{S}_{2}$.

Theorem 1.1 and other evidences force us to look for those $\mathfrak{X}$ which satisfy at least $\mathfrak{X}=\mathrm{S} \mathfrak{X}=\mathrm{QX}$ and hence this assumption will be done from now on until the end of the paper. 
Some general facts from $[14,16,17]$ will help us to have a systematic treatment of the subject in terms of class operators. Firstly, we always have

$$
\mathfrak{F} \subseteq \check{\mathfrak{C}} \subseteq \mathfrak{S}_{2} \mathfrak{F}, \quad \mathfrak{F} \subseteq \mathfrak{Z}^{\infty} \mathfrak{F} \subseteq \mathfrak{S}_{2} \mathfrak{F}, \quad \check{\mathfrak{C}} \cap \mathfrak{Z}^{\infty} \mathfrak{F}=\mathfrak{F} .
$$

Moreover, given $r \geq 1$, we may define the class operator L, by

$$
\mathrm{L} \mathfrak{X}=\left(G \mid\left\langle g^{\prime}, g_{2}, \ldots, g_{r}\right\rangle \in \mathfrak{X}, \forall g^{\prime}, g_{2}, \ldots g_{r} \in G\right),
$$

called local operator for $\mathfrak{X}$ and, immediately, LF turns out to be the class of all locally finite groups. Another important class operator is $\mathrm{H}$, defined by

$$
\mathrm{HX}=(G \mid M / N \in \mathfrak{X}, \forall N \triangleleft G, \exists M \text { with } N \subset M \triangleleft G),
$$

called extension operator. It associates to $\mathfrak{X}$ the class of hyper- $\mathfrak{X}-$ groups, defined above. Note that a hyper- $\mathfrak{X}$-group is characterized by the fact that each nontrivial homomorphic image has a normal subgroup belonging to $\mathfrak{X}$. Hyperfinite groups $\mathrm{HF}$ and hypercyclic groups $\mathrm{H} \mathfrak{Z}$ are remarkable examples.

Finally, we may define the class operator $\mathrm{C}$ by

$$
\mathfrak{X C}=\left(G \mid G / C_{G}\left(\langle x\rangle^{G}\right) \in \mathfrak{X}, \forall x \in G\right) .
$$

The class $\mathfrak{F} \mathrm{C}$ is very well known, and also $\mathfrak{Z}^{\infty} \mathfrak{F C}, \check{\mathfrak{C} C}, \mathfrak{S}_{2} \mathfrak{F} \mathrm{C}$ have been largely studied (see the references in [17] or also $[14, \S 14.5]$ ).

It may be useful to note that

(1.3) $\mathrm{S} \mathrm{L} \mathfrak{X}=\mathrm{Q} \mathrm{L} \mathfrak{X}=\mathrm{L} \mathfrak{X}, \quad \mathrm{S} H \mathfrak{X}=\mathrm{Q} H \mathfrak{X}=\mathrm{H} \mathfrak{X}$ and $\mathrm{S} \mathfrak{X C}=\mathrm{Q} \mathfrak{X C}=\mathfrak{X C}$ and that

$$
\mathfrak{X} \subseteq \mathrm{L} \mathfrak{X}, \mathfrak{X} \subseteq \mathrm{H} \mathfrak{X} \text { and } \mathfrak{X} \subseteq \mathfrak{X C} .
$$

In particular, (1.3) and (1.4) hold true for $\mathfrak{F}, \mathfrak{Z}^{\infty}, \mathfrak{Z}^{\infty} \mathfrak{F}, \check{\mathfrak{C}}, \mathfrak{S}_{2}, \mathfrak{S}_{2} \mathfrak{F}, \mathfrak{N}, \mathfrak{S}$.

To the best of our knowledge, $\mathrm{NT} \mathrm{LF}=\mathrm{L} \mathfrak{F}$ can be found in literature. The proofs of this and of most of the results of Theorem 1.1 actually use the fact that the class of groups is closed under forming extensions. But, a priori, $\mathfrak{X}$ might not satisfy this assumption. Then the following questions are open.

Question 1.2. For which $\mathfrak{X}, \mathrm{NT}$ is closed with respect to L $\mathfrak{X}$ or $\mathrm{H} \mathfrak{X}$ or $\mathfrak{X C}$ ? And, are there any connections among all these conditions?

In Section 2 we will study the above questions as well as (1.2). Homological machineries may be avoided, generalizing the methods of Thomas in [19].

\section{Results}

If $\phi: H \rightarrow H^{\phi}$ is an isomorphism (and $G$ and $H$ act compatibly upon each other by conjugation), it is possible to define the overgroup

$$
\begin{gathered}
\eta(G, H)=\left\langle G, H^{\phi}\right|{ }^{g^{\prime}}\left[g, h^{\phi}\right]=\left[{ }^{g^{\prime}} g,\left(g^{\prime} h\right)^{\phi}\right],^{h^{\prime}}\left[g, h^{\phi}\right]^{\phi}=\left[{ }^{h^{\prime}} g,\left(h^{\prime} h\right)^{\phi}\right], \\
\left.\forall g, g^{\prime} \in G, h, h^{\prime} \in H\right\rangle,
\end{gathered}
$$

which turns out to contain $G$ and $H$ via the canonical inclusions

$$
\iota_{G}: G \rightarrow \eta(G, H) \text { and } \iota_{H}: H \rightarrow \eta(G, H) .
$$


Details can be found in $[9,12,15]$. If one looks at $G$ and $H$ as normal subgroups of a bigger group $M$, then the following argument may be applied (see for instance [9, p. 23]). Denote with $X_{G}$ a generating set for $G$ and with $X_{H}$ one for $H$ and let $X=X_{G} \cup X_{H}$. Now denote by $\widetilde{X}_{G}$ a generating set of $G$, closed under conjugation by elements of $X$, and by $\widetilde{X}_{H}$ the corresponding for $H$. By $[9,19]$, we have that

where

$$
\eta(G, H) \simeq(G * H) / K
$$

$$
K=\left\langle x[g, h] x^{-1}\left[{ }^{x} g,{ }^{x} h\right] \mid x \in X, g \in \widetilde{X}_{G}, h \in \widetilde{X}_{H}\right\rangle^{G * H} .
$$

The next lemma summarizes most of the previous information.

Lemma 2.1. Let $G$ and $H$ be two normal subgroups (acting compatibly and by conjugation upon each other) of a group $M$. Then

(i) $((G \otimes H) \rtimes H) \rtimes G \simeq \eta(G, H)$;

(ii) $G \otimes H \simeq G^{\eta(G, H)} \cap H^{\eta(G, H)}$;

(iii) $D_{H}(G)$ is isomorphic to a homomorphic image of $G \otimes H$.

Proof. (i) and (ii) can be found in [19]. It remains to prove (iii). The following sequence describes $G \otimes H$ as a central extension of $\nabla(G \cap H)$ by $D_{H}(G)$

$$
1 \longrightarrow \operatorname{ker} \kappa \stackrel{\iota}{\longrightarrow} G \otimes H \stackrel{\kappa}{\longrightarrow} D_{H}(G) \longrightarrow 1
$$

so that $G \otimes H /$ ker $\kappa \simeq D_{H}(G)$, that is, $D_{H}(G)$ is isomorphic to the homomorphic image $G \otimes H / \operatorname{ker} \kappa$ of $G \otimes H$.

The reader may find different proofs of Lemma 2.1(i) and (ii) also in [12]. Now we may reformulate for our aims Theorem 1.1.

Theorem 2.2. Let $G$ and $H$ be two normal subgroups (acting compatibly and by conjugation upon each other) of a group $M$ containing $\eta(G, H)$. If $G, H \in \mathfrak{X}$, then $G \otimes H \in \mathfrak{X}$.

Proof. From Lemma 2.1(ii) we have that

$$
G \otimes H \simeq G^{\eta(G, H)} \cap H^{\eta(G, H)} \leq G^{M} \cap H^{M}=G \cap H .
$$

This means that $G \otimes H$ is isomorphic to a subgroup of $G \cap H \in \mathfrak{X}$ and, having in mind that $\mathrm{S} \mathfrak{X}=\mathrm{Q} \mathfrak{X}=\mathfrak{X}$, we may conclude that $G \otimes H \in \mathfrak{X}$.

Some consequences are listed below.

Corollary 2.3. In the hypotheses of Theorem 2.2, if $G=H \in \mathfrak{X}$, then $\mathrm{NTX}=$ $\mathfrak{X}$. In particular,

(i) $\mathrm{NT} \mathrm{LF}=\mathrm{L} \mathfrak{F}, \mathrm{NT} \mathrm{L}^{\infty}=\mathrm{L} \mathfrak{Z}^{\infty}, \mathrm{NT} \mathrm{L} \check{\mathfrak{C}}=\mathrm{L} \check{\mathfrak{C}}, \mathrm{NT} \mathrm{LS}_{2}=\mathrm{LS}_{2}$, $\mathrm{NT}$ LS $=\mathrm{LS}, \mathrm{NT}$ LN $=\mathrm{LN}$.

(ii) $\mathrm{NT} \mathrm{H} \mathfrak{F}=\mathrm{H} \mathfrak{F}, \mathrm{NT} \mathrm{H}^{\infty}=\mathrm{H}^{\infty}, \mathrm{NT} \mathrm{H \check {C }}=\mathrm{H \check {C }}, \mathrm{NT} \mathrm{HS}_{2}=\mathrm{HS}_{2}$, $\mathrm{NT} \mathrm{HS}=\mathrm{HS}$, $\mathrm{NT} \mathrm{HN}=\mathrm{HN}$.

(iii) $\mathrm{NT} \mathfrak{F C}=\mathfrak{F C}, \mathrm{NT} \mathfrak{Z}^{\infty} \mathfrak{F} \mathrm{C}=\mathfrak{Z}^{\infty} \mathfrak{F} \mathrm{C}, \mathrm{NT} \check{\mathfrak{C C}}=\check{\mathfrak{C C}}, \mathrm{NT} \mathfrak{S}_{2} \mathfrak{F} \mathrm{C}=\mathfrak{S}_{2} \mathfrak{F} \mathrm{C}$.

(iv) $\mathrm{NT} \mathfrak{P}=\mathfrak{P}$. 
Proof. The main statement is clearly an application of Theorem 2.2. Now, from (1.3) and knowing that $\mathrm{SP}=\mathrm{QP}=\mathfrak{P}$, (i), (ii), (iii) and (iv) follow.

We make an observation on the argument of the proof of Theorem 2.2.

Remark 2.4. Some of the proofs of the results summarized in Theorem 1.1 use the fact that the class of groups (which is involved) is closed under extensions. This is not used in the proof of Theorem 2.2 .

Another result similar to Theorem 2.2 is the following.

Theorem 2.5. Let $G$ and $H$ be two normal subgroups (acting compatibly and by conjugation upon each other) of a group $M$ containing $\eta(G, H)$. If $G$ and $H$ are finitely presented and $G \cap H$ has finite index in $G$ (or in $H$ ), then $G \otimes H$ is finitely presented.

Proof. From a well-known result of P. Hall [14, 14.1.3], we may deduce that finite index subgroups of finitely presented groups are finitely presented. Now $G \cap H$ has finite index in $G$ (or $H$ ) and so it is finitely presented. From Lemma 2.1(ii) we have

$$
G \otimes H \simeq G^{\eta(G, H)} \cap H^{\eta(G, H)}=G \cap H,
$$

and then $G \otimes H$ is finitely presented too. The result follows.

Remark 2.6. Higman's Group $\langle x, y, z, t| x^{-1} y x=y^{2}, y^{-1} z y=z^{2}, z^{-1} t z=$ $\left.t^{2}, t^{-1} x t=x^{2}\right\rangle$ (see $[14,3.2 .9$ at p. 77 and 6.4 .15 at p. 184]) shows that there exist finitely presented groups with subgroups and quotients which are not finitely presented. This means that the property of being finitely presented is not closed with respect to forming subgroups and quotients. Then the assumption that $G \cap H$ has finite index in $G$ cannot be omitted in Theorem 2.5 .

Remark 2.7. It is important to recall that in [5] Brown and Loday describe the role of the nonabelian tensor product in algebraic topology (see also $[1,11]$ ). They showed that the third homotopy group of the suspension of an EilenbergMacLane space $K(G, 1)$ satisfies the condition $\pi_{3} S K(G, 1) \simeq J_{2}(G)$. Also, the nonabelian tensor product is used to describe the third relative homotopy group of a triad as a (nonabelian) tensor product of the second homotopy groups of appropriate subspaces. More specifically, let a CW-complex $X$ be the union $X=A \cup B$ of two path connected CW-subcomplexes $A$ and $B$ whose intersection $C=A \cap B$ is path connected. Suppose that the canonical homomorphisms $\pi_{1}(C) \rightarrow \pi_{1}(A)$ and $\pi_{1}(C) \rightarrow \pi_{1}(B)$ are surjective. Then, one can see that $\pi_{3}(X, A, B) \simeq \pi_{2}(A, C) \otimes \pi_{2}(B, C)$, where the groups $\pi_{2}(A, C)$ and $\pi_{2}(B, C)$ act upon each another via $\pi_{1}(C)$.

In virtue of the previous remark, it is interesting to see whenever there exist geometric or topological conditions which are closed under the formation of the nonabelian tensor square. An example comes from an asymptotic invariant of 
discrete groups (i.e. a well-defined property of groups which is invariant under quasi-isometries $[8,13])$ of topological nature, due to Brick and Mihalik $[2,18]$.

Definition 2.8 (See $[2,18]$ ). The simply connected non-compact complex $X$ is qsf (i.e., quasi simply filtered) if for any connected finite subcomplex $C \subset X$ there exists a finite simply connected complex $K$ and a cellular map $f: K \rightarrow X$ so that $C \subset f(K)$ and $\left.f\right|_{f^{-1}(C)}: f^{-1}(C) \rightarrow C$ is a cellular homeomorphism. A finitely presented group $G$ is $q s f$ if the universal covering of the presentation 2-complex associated to one of its presentations is qsf.

Some crucial properties of the qsf condition are the following.

Lemma 2.9 (See $[2,3]$ ). Let $G$ be a finitely presented group.

(i) $G$ is qsf if and only if a finite index subgroup $H$ of $G$ is qsf.

(ii) If $G=A *_{C} B$ is the amalgamated product of two finitely presented groups $A$ and $B$ over a common finitely generated subgroup $C$, then $G$ is qsf provided so are $A$ and $B$.

(iii) Assume that $1 \rightarrow A \rightarrow G \rightarrow B \rightarrow 1$ is a short exact sequence of infinite finitely presented groups. If $A$ and $B$ are qsf, then $G$ is qsf.

Corollary 2.10. In the hypotheses of Theorem 2.5, if $G$ and $H$ are two infinite qsf groups, then $G \otimes H$ is qsf. Moreover, if $\eta(G, H)$ is finitely presented, then it is qsf.

Proof. From Theorem 2.5 we may conclude that $G \otimes H$ is finitely presented. Lemma 2.1(ii) implies that $G \otimes H \simeq G^{\eta(G, H)} \cap H^{\eta(G, H)}=G \cap H \leq G$.

The subgroup $G \cap H$ is an infinite finitely presented group since it is a finite index finitely presented subgroup of $G$ (by the aforementioned [14, 14.1.3]). Since finite index subgroups of qsf groups are qsf by Lemma 2.9(i), $G \cap H$ is qsf too (because it has finite index in $G$ (or in $H$ ) which is qsf). It follows that $G \otimes H$ is qsf.

Assume now that $\eta(G, H)$ is finitely presented. In order to prove that $\eta(G, H)$ is qsf, consider the short exact sequence $1 \rightarrow G \otimes H \rightarrow(G \otimes H) \rtimes H \rightarrow$ $H \rightarrow 1$, which implies that $(G \otimes H) \rtimes H$ is qsf by Lemma 2.9 (iii) and by the fact that we have just proved that $G \otimes H$ is infinite and qsf. Similarly, the short exact sequence $1 \rightarrow(G \otimes H) \rtimes H \rightarrow((G \otimes H) \rtimes H) \rtimes G \rightarrow G \rightarrow 1$ implies that $((G \otimes H) \rtimes H) \rtimes G$ is qsf by Lemma 2.9 (iii) and by the fact $(G \otimes H) \rtimes H$ is qsf. From Lemma 2.1(i) we conclude that $\eta(G, H)$ is qsf.

\section{References}

[1] W. A. Bogley and N. D. Gilbert, The homology of Peiffer products of groups, New York J. Math. 6 (2000), 55-71.

[2] S. G. Brick and M. L. Mihalik, The QSF property for groups and spaces, Math. Z. 220 (1995), no. 2, 207-217.

[3] Group extensions are quasi-simply-filtrated, Bull. Austral. Math. Soc. 50 (1994), no. $1,21-27$.

[4] R. Brown, D. L. Johnson, and E. F. Robertson, Some computations of nonabelian tensor products of groups, J. Algebra 111 (1987), no. 1, 177-202. 
[5] R. Brown and J.-L. Loday, Van Kampen theorems for diagrams of spaces, Topology 26 (1987), no. 3, 311-335.

[6] A. Erfanian, F. G. Russo, and N. H. Sarmin, Some considerations on the nonabelian tensor square of crystallographic groups, Asian-Eur. J. Math. 4 (2011), no. 2, 271-282.

[7] A. Erfanian, N. M. Mohd Ali, S. Rashid, and N. H. Sarmin, On the nonabelian tensor square and capability of groups of order $p^{2} q$, Arch. Math. (Basel) 97 (2011), no. 4, 299-306.

[8] L. Funar and D. E. Otera, On the wgsc and qsf tameness conditions for finitely presented groups, Groups Geom. Dyn. 4 (2010), no. 3, 549-596.

[9] N. Gilbert and P. Higgins, The nonabelian tensor product of groups and related constructions, Glasgow Math. J. 31 (1989), no. 1, 17-29.

[10] T. Hannebauer, On non-abelian tensor squares of linear groups, Arch. Math. (Basel) 55 (1990), no. 1, 30-34.

[11] N. Inassaridze, Nonabelian tensor products and nonabelian homology of groups, J. Pure Appl. Algebra 112 (1996), no. 2, 191-205.

[12] I. Nakaoka, Non-abelian tensor products of solvable groups, J. Group Theory 3 (2000), no. 2, 157-167.

[13] D. E. Otera and F. G. Russo, On the WGSC property in some classes of groups, Mediterr. J. Math. 6 (2009), no. 4, 501-508.

[14] D. J. Robinson, A Course in the Theory of Groups, Springer, Berlin, 1982.

[15] N. R. Rocco, On a construction related to the nonabelian tensor square of a group, Bol. Soc. Brasil. Mat. (N.S.) 22 (1991), no. 1, 63-79.

[16] F. G. Russo, Nonabelian tensor product of soluble minimax groups, Proceedings of Computational Group Theory and Cohomology (Harlaxton, 2008), 179-182, Contemporary Mathematics, American Mathematical Society, New York. 2010.

[17] _ A generalization of groups with many almost normal subgroups, Algebra Discrete Math. 9 (2010), no. 1, 79-85.

[18] J. R. Stallings, Brick's quasi-simple filtrations for groups and 3-manifolds, Procedings of Geometric Group Theory, Vol. 1 (Sussex, 1991), 188-203, London Math. Soc. Lecture Note Ser. 181, Cambridge University Press, Cambridge. 1993.

[19] V. Z. Thomas, The non-abelian tensor product of finite groups is finite: a homology-free proof, Glasg. Math. J. 52 (2010), no. 3, 473-477.

[20] M. P. Visscher, On the nilpotency class and solvability length of nonabelian tensor product of groups, Arch. Math. (Basel) 73 (1999), no. 3, 161-171.

Daniele Ettore Otera

DÉPARTEMENT DE MAThÉMATIQUe

Université PARIS Sud 11

F-91405 ORSAY CEDEX, FranCE

E-mail address: daniele.otera@gmail.com

Francesco G. Russo

DEIM

Universitá Degli StUdi di PALERMO

viale Delle Scienze, Edificio 8, 90128, Palermo, Italy

AND

Department of MATHEMATiCS

Universiti TeKNologi MALAYsia

SkUdAi, 81310, Johor, MALAYSiA

E-mail address: francescog.russo@yahoo.com 


\section{Corrado TANASI}

Dipartimento di Matematica e InFormatica

Universitá degli Studi di Palermo

Via Archirafi 34, 90123, Palermo, Italy

E-mail address: corrado.tanasi@unipa.it 